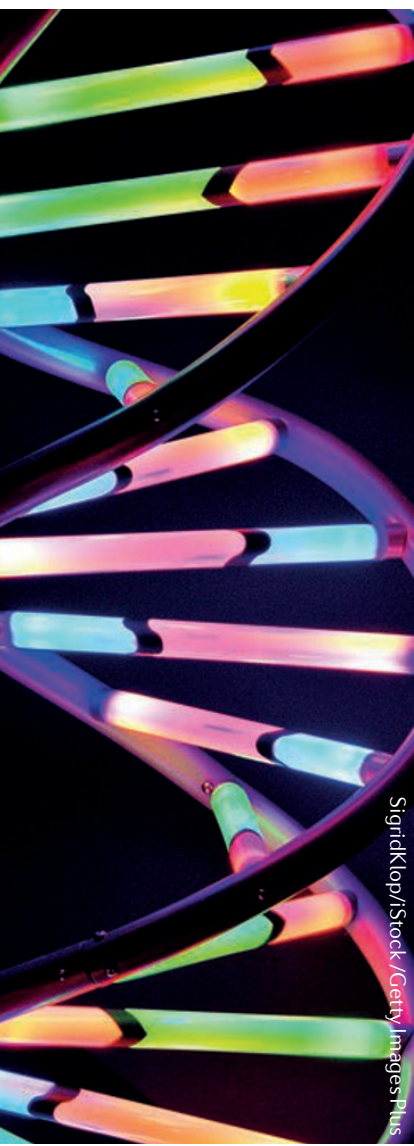

POLYCYSTIC KIDNEY DISEASE

\section{PMM2 mutation causes PKD and hyperinsulinism}

Researchers have identified a previously undescribed genetic disorder that presents with hyperinsulinaemic hypoglycaemia (HI) and polycystic kidney disease (PKD) in childhood (HIPKD). They report that this disease is caused by a promoter mutation in PMM2, which encodes the $\mathrm{N}$-glycosylation enzyme phosphomannomutase 2 .

The coexistence of PKD and $\mathrm{HI}$ - two rare diseases - in 17 children from 11 unrelated families suggested the presence of a single disorder, explains researcher Detlef Bockenhauer. To identify the genetic basis of this disorder, Bockenhauer and colleagues performed whole-genome linkage analysis in five affected families. They identified a single significant locus of 2.3Mb on chromosome 16p.13.2. This region contains 14 genes. "The logarithm of odds score was 6.5 which meant the odds were $>3,000,000$ variant was in this region," explains Bockenhauer. "However, we struggled to identify a common genetic basis of HIPKD until we started to look at the non-coding regions. We realised that all affected patients shared a variant in the promoter of $P M M 2$, either homozygously or in trans with PMM2 coding mutations."

Interestingly, biallelic mutations in PMM2 cause congenital disorder of glycosylation type $1 \mathrm{~A}(\mathrm{CDG} 1 \mathrm{~A})$, which is characterized by severe neurologic involvement, dysmorphic features and other complications. Affected patients typically die in infancy. "Our patients, despite having biallelic PMM2 mutations, have none of these features," says Bockenhauer. "Thus we have PMM2 to one that the causative genetic pleiotropy; bialleleic coding mutations cause $\mathrm{CDG} 1 \mathrm{~A}$, whereas the promoter mutation leads to organ-specific disease, such that only kidney cells, pancreatic $\beta$-cells and potentially liver cells are affected (some patients have liver cysts and ductal plate malformation)."

The PMM2 promoter variant was associated with reduced transcriptional activity of PMM2 in human kidney and pancreatic $\beta$-cell lines. Moreover, the researchers' in silico analysis suggested that this variant might affect tissue-specific chromatin loop formation, which could underlie the organ-restricted phenotype of HIPKD.

The researchers conclude that their data are consistent with a critical role of protein glycosylation in normal insulin secretion and kidney morphology. "Our findings also provide insights into how genes can be turned on or off in a space and potentially time-specific manner," says Bockenhauer.

Ellen F. Carney

ORIGINAL ARTICLE Rubio Cabezas, O. et al.

Polycystic kidney disease with hyperinsulinemic hypoglycemia caused by a promoter mutation in phosphomannomutase 2.J.Am. Soc. Nephrol. http://dx.doi.org/10.1681/ASN.2016121312 (2017) 